\title{
Generators of Bieberbach groups with 2-generated holonomy group
}

\section{Ho Yiu Chung ${ }^{1}$}

Received: 1 August 2018 / Accepted: 8 November 2018 / Published online: 14 November 2018

(c) The Author(s) 2018

\begin{abstract}
An $n$-dimensional Bieberbach group is the fundamental group of a closed flat $n$-dimensional manifold. K. Dekimpe and P. Penninckx conjectured that an $n$-dimensional Bieberbach group can be generated by $n$ elements. In this paper, we show that the conjecture is true if the holonomy group is 2-generated (e.g. dihedral group, quaternion group or simple group) or the order of holonomy group is not divisible by 2 or 3 . In order to prove this, we show that an $n$-dimensional Bieberbach group with cyclic holonomy group of order larger than two can be generated by $(n-1)$ elements.
\end{abstract}

Keywords Crystallographic group · Bieberbach group · Generators · Cyclic group

\section{Introduction}

We first introduce the geometric definition of a crystallographic groups. A group $\Gamma$ is said to be an $n$-dimensional crystallographic group if it is a discrete subgroup of $\mathbb{R}^{n} \rtimes O(n)$, which is the group of isomotries of $\mathbb{R}^{n}$ and it acts cocompactly on $\mathbb{R}^{n}$. By The First Bieberbach Theorem, [15, Theorem 2.1], $\Gamma \cap\left(\mathbb{R}^{n} \times I\right)$ is isomorphic to $\mathbb{Z}^{n}$ and $\Gamma / \Gamma \cap\left(\mathbb{R}^{n} \times I\right)$ is a finite group called the holonomy groups of $\Gamma$. We say $\Gamma$ is an $n$-dimensional Bieberbach group if it is an $n$-dimensional torsion-free crystallographic group. In this paper, we focus on the below conjecture.

Conjecture 1.1 [8, Dekimpe-Penninckx] Let $\Gamma$ be an $n$-dimensional Bieberbach group. Then the minimum number of generators of $\Gamma$ is less than or equal to $n$.

The conjecture was solved for some special cases. For example, the conjecture is true if the holonomy group is an odd prime $p$-group (see [1]), or the holonomy group is an elementary abelian $p$-group (see [8]). On the other hand, by a computer program namely CARAT, it has been checked that the conjecture is true if the Bieberbach group has dimension less than 7 (see [5]).

There is a connection between the number of generators of Bieberbach group and the number of generators of a finite group that can act freely on an $n$-torus (see [10]). Let $G$ be

\footnotetext{
$\bowtie$ Ho Yiu Chung

hyc1g16@soton.ac.uk

1 University of Southampton, Southampton, UK
} 
a finite group. If $G$ acts freely on an $n$-torus $T^{n}$, the quotient space $T^{n} / G$ is a manifold $M$ and we get a short exact sequence as below,

$$
0 \longrightarrow \pi_{1}\left(T^{n}\right) \longrightarrow \pi_{1}(M) \longrightarrow G \longrightarrow
$$

where $\pi_{1}(M)$ is an $n$-dimensional Bieberbach group. Hence if $\pi_{1}(M)$ can be generated by $n$ elements, then the minimal number of generators of $G$ should not be larger than $n$. For instance, we know that $(\mathbb{Z} / 2 \mathbb{Z})^{n+1}$ cannot act freely on $T^{n}$ for $n \geq 1$. (see $[8,10]$ ).

Let $G$ be a group and $M$ be a $\mathbb{Z} G$-module. Throughout this paper, we denote $d(G)$ to be the minimal number of generators of $G$ and denote $r k_{G}(M)$ to be the minimal number of generators of $M$ as a $\mathbb{Z} G$-module. Our paper is divided into several sections. In Sect. 2, we give some basic definitions and some related properties of crystallographic groups. In Sect. 3, we discuss the number of generators of $\mathbb{Z} C_{m}$-module, where $C_{m}$ is a cyclic group of order $m$. In Sect. 4, we present our three main theorems. The below three theorems are our main results.

Theorem A Let $\Gamma$ be an $n$-dimensional crystallographic group with holonomy group isomorphic to $C_{m}=\left\langle g \mid g^{m}=1\right\rangle$ where $m \geq 3$.

(i) If $m$ is divisible by prime larger than 3 , then $d(\Gamma) \leq n-2$.

(ii) If $m$ is not divisible by prime larger than 3 and $\Gamma$ is torsion-free, then $d(\Gamma) \leq n-1$.

The idea of the proof of Theorem A(i) is to consider $\Gamma \cap\left(\mathbb{R}^{n} \times I\right)$ as a $\mathbb{Z} C_{p}$-module where $p$ is prime larger than 3 . We use the module structure to reduce the number of generators. For Theorem A(ii), we construct a surjective homomorphism from $\Gamma$ to $\mathbb{Z}$. Then by studying how $\mathbb{Z}$ acts on the kernel of the homomorphism, we can eliminate some redundant generators.

By Theorem A, we get two corollaries. One shows that a general $n$-dimensional Bieberbach group can be generated by $2 n$ elements. The other corollary shows an $n$-dimensional Bieberbach group with a simple group as holonomy group can be generated by $n-1$ elements.

Theorem B Let $\Gamma$ be an $n$-dimensional crystallographic group with holonomy group isomorphic to a finite group $G$,

(i) If the order of $G$ is not divisible by 2 or 3 , then $d(\Gamma) \leq n$.

(ii) If the order of $G$ is odd and divisible by 3 , then $d(\Gamma) \leq n+1$.

The idea of the proof of Theorem B is to apply results from [11] to get a relation between the number of generators of the finite group $G$ and its Sylow $p$-subgroups. Then we apply results from [1] to prove Theorem B.

Theorem C Let $\Gamma$ be an n-dimensional Bieberbach group with 2-generated holonomy group. Then $d(\Gamma) \leq n$.

The idea of the proof of Theorem $\mathrm{C}$ is to consider a Bieberbach subgroup with cyclic holonomy group. Then we apply Theorem A to get the desired bound for generators of the Bieberbach group $\Gamma$.

\section{Background}

In this section, we recall some properties of crystallographic group from $[6,15]$. Let $\Gamma$ be an $n$-dimensional crystallographic group. By The First Bieberbach Theorem, [15, Theorem 
2.1], $\Gamma \cap\left(\mathbb{R}^{n} \times I\right)$ is isomorphic to $\mathbb{Z}^{n}$ and it is the maximal abelian subgroup with finite index, where $I$ is the identity element in the orthogonal group. Therefore $\Gamma$ can be expressed as the short exact sequence

$$
0 \longrightarrow \mathbb{Z}^{n} \stackrel{\iota}{\longrightarrow} \Gamma \stackrel{p}{\longrightarrow} G \longrightarrow 1
$$

where $G$ is a finite group, $\iota: \mathbb{Z}^{n} \hookrightarrow \Gamma$ is an inclusion map which maps $e_{i}$ to $\left(e_{i}, I\right)$ where $e_{1}, \ldots, e_{n}$ are the standard basis of $\mathbb{Z}^{n}$ and $p: \Gamma \rightarrow G$ is a projection map which maps $(a, A)$ to $A$. Given such a short exact sequence, it will induce a representation $\rho: G \rightarrow G L_{n}(\mathbb{Z})$ given by $\rho(g) x=\bar{g} \iota(x) \bar{g}^{-1}$, where $x \in \mathbb{Z}^{n}$ and $\bar{g}$ is chosen arbitrarily such that $p(\bar{g})=g$. In this case, we call the group $G$ to be the holonomy group and the representation $\rho$ to be the holonomy representation of $\Gamma$. It is well known that $\rho$ is a faithful representation (see [15, Chapter 2]).

Now we are going to introduce the algebraic definition for crystallographic groups, which is equivalent to the geometric definition of crystallographic groups (see [15, Theorem 2.2]). We say $\Gamma$ is an $n$-dimensional crystallographic group if it can be expressed as the below short exact sequence

$$
0 \longrightarrow \mathbb{Z}^{n} \longrightarrow \Gamma \longrightarrow 1
$$

where $G$ is finite group and the induce representation $\rho: G \rightarrow G L_{n}(\mathbb{Z})$ is a faithful representation. Given an $n$-dimensional crystallographic group $\Gamma$, with holonomy group $G$. Every element $\gamma \in \Gamma$ can be expressed as $(a, g)$ where $a \in \mathbb{R}^{n}$ and $g \in G$. The operation in $\Gamma$ is given by $\left(a_{1}, g_{1}\right)\left(a_{2}, g_{2}\right)=\left(a_{1}+\rho\left(g_{1}\right)\left(a_{2}\right), g_{1} g_{2}\right)$, where $(a, g),(b, h) \in \Gamma$ and $\rho$ is the holonomy representation. Notice that $\Gamma$ will induce the holonomy representation $\rho: G \rightarrow G L_{n}(\mathbb{Z})$. Therefore we can consider $\Gamma \cap\left(\mathbb{R}^{n} \times I\right) \cong \mathbb{Z}^{n}$ as a $\mathbb{Z} G$-module. We denote $r k_{G}\left(\mathbb{Z}^{n}\right)$ to be the minimal number of generators of $\mathbb{Z}^{n}$ as a $\mathbb{Z} G$-module. In particular, if $G$ is a cyclic group with generator $g$ and let $\rho: G \rightarrow G L_{n}(\mathbb{Z})$ where $g \mapsto M \in G L_{n}(\mathbb{Z})$ be its matrix holonomy representation. For convenience, we denote element $(a, g) \in \Gamma$ to be $(a, M)$ and denote the $\mathbb{Z} G$-module $\mathbb{Z}^{n}$ to be $\mathbb{Z}_{M}^{n}$ to specify that the $G$-action is given by the matrix $M$. We will denote $I_{n}$ to be the identity matrix of dimension $n$ and $C_{m}$ to be a cyclic group of order $m$.

Remark 2.1 Let $\Gamma$ be an $n$-dimensional crystallographic group with holonomy group $G$, where $G$ is generated by $m$ elements namely $a_{1}, \ldots, a_{m}$. Then by sequence (1), we have the following two observations,

(i) $d(\Gamma) \leq r k_{G}\left(\mathbb{Z}^{n}\right)+d(G)$.

(ii) $\left\{\iota\left(e_{1}\right), \ldots, \iota\left(e_{n}\right), \alpha_{1}, \ldots, \alpha_{m}\right\}$ can be a generating set of $\Gamma$ where $e_{1}, \ldots, e_{n}$ are the standard basis of $\mathbb{Z}^{n}$ and $\alpha_{i}$ is chosen arbitrarily such that $p\left(\alpha_{i}\right)=a_{i}$ for all $i=$ $1, \ldots, m$.

Definition 2.2 Let $G$ be a group, $M$ be a $\mathbb{Z} G$-module and $\rho: G \rightarrow G L_{m}(\mathbb{Z})$ be the representation correspond to the $\mathbb{Z} G$-module $M$.

(i) $N$ is a submodule of $M$ if $N$ is a subgroup of $M$ which is closed under the action of ring elements.

(ii) $M$ is decomposable if $M$ is the direct sum of submodules. $M$ is indecomposable if $M$ is not decomposable.

(iii) $M$ is $\mathbb{Z}$-reducible if there exists a matrix $N \in G L_{m}(\mathbb{Z})$ such that $N \rho(g) N^{-1}=\left(\begin{array}{l}* * \\ 0 *\end{array}\right)$ for $g \in G$. $M$ is $\mathbb{Z}$-irreducible if $M$ is not $\mathbb{Z}$-reducible. 
Now, we are going to give a short introduction to the properties of holonomy representation. Let $M_{1}, \ldots, M_{k}$ be square matrices with entries in $\mathbb{Z}$, we denote $\operatorname{tri}\left(M_{1}, \ldots, M_{k}\right)$ to be matrix of form as below,

$$
\operatorname{tri}\left(M_{1}, \ldots, M_{k}\right):=\left(\begin{array}{llll}
M_{1} & & & * \\
& M_{2} & & \\
& & \ddots & \\
0 & & & M_{k}
\end{array}\right)
$$

Let $\rho: C_{m} \rightarrow G L_{n}(\mathbb{Z})$ be a faithful representation. Since $\rho$ is defined up to isomorphism, we are able to conjugate it by a suitable invertible matrix and assume $\rho(g)=\operatorname{tri}\left(A_{1}, \ldots, A_{t}\right)$ for some $t \in \mathbb{N}$ and $A_{1}, \ldots, A_{t}$ are square matrices such that $\mathbb{Z}_{A_{1}}^{\operatorname{dim}\left(A_{1}\right)}, \ldots, \mathbb{Z}_{A_{t}}^{\operatorname{dim}\left(A_{t}\right)}$ are $\mathbb{Z}$ irreducible modules and $\sum_{j=1}^{t} \operatorname{dim}\left(A_{j}\right)=n$.

Remark 2.3 Let $M=\operatorname{tri}\left(A_{1}, \ldots, A_{t}\right)$ where $A_{1}, \ldots, A_{t}$ are square matrices. Denote the order of $A_{i}$ to be $a_{i}$ for $i=1, \ldots, t$ and $m$ to be the order of $M$. Then the least common multiple of $a_{1}, \ldots, a_{t}$ equals to $m$. In particular, $m$ is divisible by $a_{i}$ for $i=1, \ldots, t$.

\section{Generators of $\mathbb{Z} C_{m}$-module}

Let $\Gamma$ be an $n$-dimensional crystallographic group with holonomy group isomorphic to $C_{m}$. We can consider $\Gamma \cap\left(\mathbb{R}^{n} \times I\right) \cong \mathbb{Z}^{n}$ as a $\mathbb{Z} C_{m}$-module. Since we can restrict the $C_{m}$-action to be a $C_{k}$-action as long as $m$ is divisible by $k$, we can also view $\mathbb{Z}^{n}$ as a $\mathbb{Z} C_{k}$-module. It is clear that $r k_{C_{m}}\left(\mathbb{Z}^{n}\right) \leq r k_{C_{k}}\left(\mathbb{Z}^{n}\right)$. The below lemma and proposition are on the number of generators of $\mathbb{Z} C_{m}$-module.

Lemma 3.1 Let $\rho: C_{p} \rightarrow G L_{n}(\mathbb{Z})$ be a faithful representation and $\mathbb{Z}^{n}$ be the correspondence $\mathbb{Z} C_{p}$-module, where $p$ is prime. Then $r k_{C_{p}}\left(\mathbb{Z}^{n}\right) \leq n-p+a$, where $a=2$ if $p \leq 19$, otherwise $a=3$.

Proof Let $g$ be the generator of $C_{p}$. Assume $\rho(g)=\operatorname{tri}\left(A_{1}, \ldots, A_{k}\right)$ where $\mathbb{Z}_{A_{1}}^{\operatorname{dim}\left(A_{1}\right)}, \cdots$, $\mathbb{Z}_{A_{k}}^{\operatorname{dim}\left(A_{k}\right)}$ are $\mathbb{Z}$-irreducible $\mathbb{Z} C_{p}$-modules. By Remark 2.3 , there exists $i \in\{1, \ldots, k\}$ such that $A_{i}$ has order $p$. By [7, Section 74] $A_{i}$ has dimension $p-1$ and the module $\mathbb{Z}_{A_{i}}^{\operatorname{dim}\left(A_{i}\right)}$ is isomorphic to an ideal in $\mathbb{Z}[\zeta]$ where $\zeta$ is a primitive $p$-root of unity. If $p \leq 19$, by [13, Section 29.1.3], the class number of $\mathbb{Z}[\zeta]$ is 1 . Therefore the module $\mathbb{Z}_{A_{i}}^{\operatorname{dim}\left(A_{i}\right)}$ is a principle ideal and it is isomorphic to $\mathbb{Z}[\zeta]$. Hence $r k_{C_{p}}\left(\mathbb{Z}_{A_{i}}^{\operatorname{dim}\left(A_{i}\right)}\right)=1$. Now assume $p>19$. Since $\mathbb{Z}[\zeta]$ is a Dedekind domain. By [13, Section 7.1-2], every ideal in a Dedekind domain can be generated by two elements. Hence $r k_{C_{p}}\left(\mathbb{Z}_{A_{i}}^{\operatorname{dim}\left(A_{i}\right)}\right) \leq 2$. Therefore we have

$r k_{C_{p}}\left(\mathbb{Z}^{n}\right) \leq n-\operatorname{dim}\left(A_{i}\right)+r k_{C_{p}}\left(\mathbb{Z}_{A_{i}}^{\operatorname{dim}\left(A_{i}\right)}\right)=n-p+1+r k_{C_{p}}\left(\mathbb{Z}_{A_{i}}^{\operatorname{dim}\left(A_{i}\right)}\right) \leq n-p+a$

where $a=2$ if $p \leq 19$, otherwise $a=3$.

Proposition 3.2 Let $\rho: C_{m} \rightarrow G L_{n}(\mathbb{Z})$ be a faithful representation and $\mathbb{Z}^{n}$ be the correspondence $\mathbb{Z} C_{m}$-module of $\rho$, where $m \geq 3$.

(i) If $m$ is divisible by prime larger than 3 , then $r k_{C_{m}}\left(\mathbb{Z}^{n}\right) \leq n-3$.

(ii) If $m$ is not divisible by prime larger than 3 , then $r k_{C_{m}}\left(\mathbb{Z}^{n}\right) \leq n-1$. 
Proof Let $m=p_{1}^{s_{1}} \cdots p_{t}^{s_{t}}$ be the prime decomposition of $m$ and assume $p_{1}<\cdots<p_{t}$. Let $g$ be the generator of $C_{m}$.

(i): Consider $H=\left\langle g^{m / p_{t}}\right\rangle \cong C_{p_{t}}$, a subgroup of $C_{m}$. We can view $\mathbb{Z}^{n}$ as a $\mathbb{Z} C_{p_{t}}$-module where the $C_{p_{t}}$-action is given by $\left.\rho\right|_{H}$. Since $\left.\rho\right|_{H}$ is a faithful representation, by Lemma 3.1, we have $r k_{C_{p_{t}}}\left(\mathbb{Z}^{n}\right) \leq n-p_{t}+a$. Since $m$ is divisible by prime larger than 3 , we have $r k_{C_{m}}\left(\mathbb{Z}^{n}\right) \leq n-3$.

(ii): We observe that $m$ is either divisible by 3 or 4 . If $m$ is divisible by 3, we consider $\mathbb{Z}^{n}$ as $\mathbb{Z} C_{3}$-module. By Lemma 3.1, we have $r k_{C_{3}}\left(\mathbb{Z}^{n}\right) \leq n-1$. Hence $r k_{C_{m}}\left(\mathbb{Z}^{n}\right) \leq n-1$. Now we assume $m$ is divisible by 4. Consider $H^{\prime}=\left\langle g^{m / 4}\right\rangle \cong C_{4}$, a subgroup of $C_{m}$. We can view $\mathbb{Z}^{n}$ as a $\mathbb{Z} C_{4}$-module by restricting the $C_{m}$-action to a $C_{4}$-action, where the $C_{4}$-action is given by $\left.\rho\right|_{H^{\prime}}$. We assume $\left.\rho\right|_{H^{\prime}}\left(g^{m / 4}\right)=\operatorname{tri}\left(M_{1}, \ldots, M_{k}\right)$ where $\mathbb{Z}_{M_{1}}^{\operatorname{dim}\left(M_{1}\right)}, \ldots, \mathbb{Z}_{M_{k}}^{\operatorname{dim}\left(M_{k}\right)}$ are $\mathbb{Z}$-irreducible $\mathbb{Z} C_{4}$-modules. By Remark 2.3, there exists $i \in\{1, \ldots, k\}$ such that $M_{i}$ is a matrix of order 4 . Let $\phi: C_{4} \rightarrow G L_{n}(\mathbb{Z})$ be the corresponding representation of $\mathbb{Z}_{M_{i}}^{\operatorname{dim}\left(M_{i}\right)}$. By [2, Section 5], there is only one faithful integral $\mathbb{Z}$-irreducible $C_{4}$-representation up to equivalence. Hence we assume $M_{i}$ is equivalent to $\left(\begin{array}{cc}0 & 1 \\ -1 & 0\end{array}\right)$. Let $y_{1}=(1,0) \in \mathbb{Z}^{2}$ and $y_{2}=(0,1) \in \mathbb{Z}^{2}$ be the standard basis of $\mathbb{Z}_{M_{i}}^{2}$. We have $\phi\left(g^{m / 4}\right) y_{2}=y_{1}$. Hence $\mathbb{Z}_{M_{i}}^{2}$ can be generated by $y_{2}$ as a $\mathbb{Z} C_{4}$-module. Since $r k_{C_{4}}\left(\mathbb{Z}_{M_{i}}^{m_{i}}\right) \leq m_{i}-1$, we have $r k_{C_{m}}\left(\mathbb{Z}^{n}\right) \leq$ $\sum_{z=1}^{k} r k_{C_{4}}\left(\mathbb{Z}_{M_{z}}^{m_{z}}\right) \leq n-1$.

\section{Main result}

Theorem A Let $\Gamma$ be an n-dimensional crystallographic group with holonomy group isomorphic to $C_{m}=\left\langle g \mid g^{m}=1\right\rangle$ where $m \geq 3$.

(i) If $m$ is divisible by prime larger than 3 , then $d(\Gamma) \leq n-2$.

(ii) If $m$ is not divisible by prime larger than 3 and $\Gamma$ is torsion-free, then $d(\Gamma) \leq n-1$.

Proof (i): By Remark 2.1, we have $d(\Gamma) \leq r k_{C_{m}}\left(\mathbb{Z}^{n}\right)+1$. Since $m$ is divisible by prime larger than 3, by Proposition 3.2, we have $r k_{C_{m}}\left(\mathbb{Z}^{n}\right) \leq n-3$. Therefore we have $d(\Gamma) \leq n-2$.

(ii): By Remark 2.1, let $\Gamma=\left\langle\iota\left(e_{1}\right), \ldots, \iota\left(e_{n}\right), \alpha\right\rangle$, where $e_{1}, \ldots, e_{n}$ are the standard basis of $\mathbb{Z}^{n}$ and $p(\alpha)=g$. By [12, Proposition 1.4] and [15, Lemma 5.2], we have $b_{1}(\Gamma)=r k\left(\left(\mathbb{Z}^{n}\right)^{C_{m}}\right)$. It is well known that $b_{1}(\Gamma) \neq 0$ (see [15, Example 4.1]). Let $k=b_{1}(\Gamma)>0$. Without loss of generality, every element of $\Gamma$ can be expressed as $\left(a, \operatorname{tri}\left(M, I_{k}\right)\right)$ where $a \in \mathbb{R}^{n}$ and $M$ is a square matrix of dimension $n-k$. In particular, let $\alpha=\left(x, \operatorname{tri}\left(A, I_{k}\right)\right)$ where $x=\left(x_{1}, \ldots, x_{n}\right) \in \mathbb{R}^{n}$ and $A$ is a square matrix of dimension $n-k$ which do not fix any non-trivial elements. In other words, $A u=u$ if and only if $u=0$ for $u \in \mathbb{R}^{n-k}$. First we assume $x_{n-k+1}=\cdots=x_{n}=0$. Let $v:=\left(x_{1}, \ldots, x_{n-k}\right) \in \mathbb{R}^{n-k}$. By simple calculations, we get $\alpha^{m}=\left(\left(\sum_{s=0}^{m-1} A^{s} v, 0, \ldots, 0\right), I_{n}\right)$. Since $A\left(\sum_{s=0}^{m-1} A^{s} v\right)=\sum_{s=0}^{m-1} A^{s} v$, we have $\sum_{s=0}^{m-1} A^{s} v=0$. There is a contradiction because $\alpha^{m}=\left(0, I_{n}\right)$. Therefore there exists $i \in\{n-k+1, \ldots, n\}$ such that $x_{i}=\frac{q}{z} \neq 0 \in \mathbb{Q}$. Define $f: \Gamma \rightarrow \mathbb{Z}$ where it maps $\left(\left(y_{1}, \ldots, y_{n}\right), \operatorname{tri}\left(M, I_{k}\right)\right) \in \Gamma$ to $z y_{i} \in \mathbb{Z}$. Hence we have $f(\alpha)=q, f\left(\iota\left(e_{i}\right)\right)=z$ and $f\left(\iota\left(e_{j}\right)\right)=0$ for all $j \neq i$. We claim that $f$ is a surjective homomorphism. Let $\gamma_{1}=\left(\left(m_{1}, \ldots, m_{n}\right), \operatorname{tri}\left(M_{1}, I_{k}\right)\right) \in \Gamma$ and $\gamma_{2}=\left(\left(\bar{m}_{1}, \ldots, \bar{m}_{n}\right), \operatorname{tri}\left(M_{2}, I_{k}\right)\right) \in \Gamma$. By simple calculation, we get $\gamma_{1} \gamma_{2}=\left(\left(*, \ldots, *, m_{n-k+1}+\bar{m}_{n-k+1}, \ldots, m_{n}+\right.\right.$ $\left.\left.\bar{m}_{n}\right), \operatorname{tri}\left(M_{1} M_{2}, I_{k}\right)\right)$. Hence we have $f\left(\gamma_{1}\right)+f\left(\gamma_{2}\right)=f\left(\gamma_{1} \gamma_{2}\right)$. Therefore $f$ is a 
homomorphism. Notice that $q$ and $z$ are coprime, there exists integers $\sigma$ and $\tau$ such that $\sigma q+\tau z=1$. Hence we have $f\left(\alpha^{\sigma} \iota\left(e_{i}\right)^{\tau}\right)=1$. Therefore $f$ is surjective. Observe that $\operatorname{ker}(f)=\left\langle\iota\left(e_{1}\right), \ldots, \iota\left(\hat{e}_{i}\right), \ldots, \iota\left(e_{n}\right)\right\rangle \cong \mathbb{Z}^{n-1}$. We have the below short exact sequence

$$
0 \longrightarrow \operatorname{ker}(f) \cong \mathbb{Z}^{n-1} \longrightarrow \Gamma \longrightarrow \mathbb{f} \longrightarrow \mathbb{Z} \longrightarrow
$$

By [4, Chapter IV, Section 1], such short exact sequence will induce a representation $\rho: \mathbb{Z} \rightarrow G L_{n-1}(\mathbb{Z})$ given by $\rho(x) e_{j}=\bar{x}_{\iota}\left(e_{j}\right) \bar{x}^{-1}$ where $f(\bar{x})=x$ for all $j \neq i$. Consider the restriction $\bar{\rho}:=\left.\rho\right|_{q \mathbb{Z}}: q \mathbb{Z} \rightarrow G L_{n-1}(\mathbb{Z})$. We claim that $\operatorname{ker}(\bar{\rho})=$ $m q \mathbb{Z}$. Let $q x \in \operatorname{ker}(\bar{\rho})$ for any $x \in \mathbb{Z}$. We have $e_{j}=\bar{\rho}(q x) e_{j}=\alpha^{x} \iota\left(e_{j}\right) \alpha^{-x}=$ $p\left(\alpha^{x}\right) e_{j}$ for all $j \neq i$. Hence $p\left(\alpha^{x}\right)$ needs to be an identity matrix. Therefore $x$ is multiple of $m$ or $x=0$. Hence $\operatorname{ker}(\bar{\rho}) \subseteq m q \mathbb{Z}$. Since $p\left(\alpha^{m}\right)$ is an identity matrix, $\rho(m q x)\left(e_{j}\right)=\alpha^{m x} \iota\left(e_{j}\right) \alpha^{-m x}=p\left(\alpha^{m x}\right) e_{j}=e_{j}$ for all $j \neq i$ and $x \in \mathbb{Z}$. Hence $m q \mathbb{Z} \subseteq \operatorname{ker}(\bar{\rho})$. Therefore we have $\operatorname{ker}(\bar{\rho})=m q \mathbb{Z}$. Now we can obtain a faithful representation $\phi: a \mathbb{Z} / m a \mathbb{Z} \rightarrow G L_{n-1}(\mathbb{Z})$ given by $\phi(\bar{x})=\bar{\rho}(x)$ where $x$ is the representative of $\bar{x} \in a \mathbb{Z} / m a \mathbb{Z}$. Hence we can view $\mathbb{Z}^{n-1}$ as a $\mathbb{Z} C_{m}$-module with faithful $C_{m}$-representation. By Proposition 3.2, $\mathbb{Z}^{n-1}$ can be generated by $n-2$ elements. By (3), we have $d(\Gamma) \leq r k_{C_{m}}\left(\mathbb{Z}^{n-1}\right)+1 \leq n-1$.

The corollary below gives the general bound on the number of generators of general Bieberbach groups.

Corollary 4.1 Let $\Gamma$ be an n-dimensional Bieberbach group with holonomy group $G$. Then $d(\Gamma) \leq 2 n$.

Proof Let $|G|=p_{1}^{s_{1}} \cdots p_{k}^{s_{k}}$ be the prime decomposition of order of $G$. By [11, Theorem A], we have

$$
d(G) \leq \max _{1 \leq i \leq k} d\left(P_{i}\right)+1
$$

where $P_{i}$ is the Sylow $p_{i}$-subgroup of $G$ for $i=1, \ldots, k$. We fix $j \in\{1, \ldots, k\}$ such that $d\left(P_{j}\right)=\max _{1 \leq i \leq k} d\left(P_{i}\right)$. We first assume $p_{j} \geq 3$. We can consider $\Gamma \cap\left(\mathbb{R}^{n} \times I\right) \cong \mathbb{Z}^{n}$ as a $\mathbb{Z} P_{j}$-module. By $\left[1\right.$, Theorem A], we have $d\left(P_{j}\right)+r k_{P_{j}}\left(\mathbb{Z}^{n}\right) \leq n$. Hence we have $d(\Gamma) \leq d(G)+1+r k_{P_{j}}\left(\mathbb{Z}^{n}\right) \leq n+1$. Now we assume $p_{j}=2$. If $G$ is a 2 -group, then by [1, Theorem A], we have $d(\Gamma) \leq 2 n$. If $G$ is not a 2-group, then there exists $g \in G$ such that $g$ has order $p \geq 3$. Hence we can consider $\mathbb{Z}^{n}$ as a $\mathbb{Z} C_{p}$-module. By Lemma 3.1, we have $r k_{C_{p}}\left(\mathbb{Z}^{n}\right) \leq n-1$. By [1, Proposition 2.2], we have $d\left(P_{j}\right) \leq n$. Hence we have $d(\Gamma) \leq 2 n$.

Corollary 4.2 Let $\Gamma$ be an $n$-dimensional Bieberbach group with holonomy group $G$, where $G$ is a simple group but not $C_{2}$. Then $d(\Gamma) \leq n-1$.

Proof By Remark 2.1, we have $d(\Gamma) \leq d(G)+r k_{G}\left(\mathbb{Z}^{n}\right)$. If $G$ is a cyclic group of odd prime order, then by Theorem A, we have $d(\Gamma) \leq n-1$. If $G$ is not cyclic, by Burnside's Theorem, [9, Page 886], there exists a prime $p \geq 5$ such that the order of $G$ is divisible by $p$. So we can view $\mathbb{Z}^{n}$ as a $\mathbb{Z} C_{p}$-module. By Lemma 3.1, we have $r k_{C_{p}}\left(\mathbb{Z}^{n}\right) \leq n-p+a \leq n-3$, where $a=2$ if $p \leq 19$, otherwise $a=3$. By [3, Theorem B], we have $d(G) \leq 2$. Hence we have $d(\Gamma) \leq d(G)+r k_{G}\left(\mathbb{Z}^{n}\right) \leq 2+r k_{C_{p}}\left(\mathbb{Z}^{n}\right) \leq n-1$. 
The rest of the paper will present the proof of Theorems B and C.

Theorem B Let $\Gamma$ be an $n$-dimensional crystallographic group with holonomy group isomorphic to a finite group $G$,

(i) If the order of $G$ is not divisible by 2 or 3 , then $d(\Gamma) \leq n$.

(ii) If the order of $G$ is odd and divisible by 3 , then $d(\Gamma) \leq n+1$.

Proof Let $|G|=p_{1}^{s_{1}} \cdots p_{k}^{s_{k}}$ be the prime decomposition of the order of $G$, where $p_{1}<$ $\cdots<p_{k}$.

(i): First, we want to calculate the number of generators of the holonomy group $G$. By [11, Theorem A], we have

$$
d(G) \leq \max _{1 \leq i \leq k} d\left(P_{i}\right)+1
$$

where $P_{i}$ is the Sylow $p_{i}$-subgroup of $G$ for $i=1, \ldots, k$. We fix $j \in\{1, \ldots, k\}$ such that $d\left(P_{j}\right)=\max _{1 \leq i \leq k} d\left(P_{i}\right)$. Let $\rho: G \rightarrow G L_{n}(\mathbb{Z})$ be the holonomy representation for $\Gamma$. By definition, $\rho$ is a faithful representation. Therefore $P_{i}$ acts faithfully on $\mathbb{Z}^{n}$. By $[1$, Proposition 2.2], we have

$$
d(G) \leq \frac{n-r k\left(\left(\mathbb{Z}^{n}\right)^{P_{j}}\right)}{p_{j}-1}+1
$$

Now, we consider the lattice part. We can view $\Gamma \cap\left(\mathbb{R}^{n} \times I\right) \cong \mathbb{Z}^{n}$ as a $\mathbb{Z} P_{j}$-module. By [1, Proposition 2.5], we have

$$
r k_{P_{j}}\left(\mathbb{Z}^{n}\right) \leq \frac{(a-1)\left(n-r k\left(\mathbb{Z}^{n}\right)^{P_{j}}\right)}{p_{j}-1}+r k\left(\mathbb{Z}^{n}\right)^{P_{j}}
$$

where $a=2$ if $p_{j} \geq 19$, otherwise $a=3$. Therefore we have

$$
\begin{aligned}
d(\Gamma) \leq d(G)+r k_{P_{j}}\left(\mathbb{Z}^{n}\right) & \leq \frac{n-r k\left(\left(\mathbb{Z}^{n}\right)^{P_{j}}\right)}{p_{j}-1}+1+\frac{(a-1)\left(n-r k\left(\mathbb{Z}^{n}\right)^{P_{j}}\right)}{p_{j}-1}+r k\left(\mathbb{Z}^{n}\right)^{P_{j}} \\
& =\frac{a\left(n-r k\left(\mathbb{Z}^{n}\right)^{P_{j}}\right)}{p_{j}-1}+r k\left(\mathbb{Z}^{n}\right)^{P_{j}}+1
\end{aligned}
$$

We need to show

$$
\frac{a\left(n-r k\left(\mathbb{Z}^{n}\right)^{P_{j}}\right)}{p_{j}-1}+r k\left(\mathbb{Z}^{n}\right)^{P_{j}}+1 \leq n
$$

We have

$$
\begin{aligned}
& \frac{a\left(n-r k\left(\mathbb{Z}^{n}\right)^{P_{j}}\right)}{p_{j}-1}+r k\left(\mathbb{Z}^{n}\right)^{P_{j}}+1 \leq n \\
\Longleftrightarrow & a n-a \cdot r k\left(\mathbb{Z}^{n}\right)^{P_{j}}+\left(p_{j}-1\right) r k\left(\mathbb{Z}^{n}\right)^{P_{j}}+p_{j}-1 \leq n\left(p_{j}-1\right) \\
\Longleftrightarrow & \left(p_{j}-1-a\right) r k\left(\mathbb{Z}^{n}\right)^{P_{j}} \leq\left(p_{j}-1-a\right) n-\left(p_{j}-1\right) \\
\Longleftrightarrow & r k\left(\mathbb{Z}^{n}\right)^{P_{j}} \leq n-\frac{p_{j}-1}{p_{j}-1-a}=n-1-\frac{a}{p_{j}-1-a}
\end{aligned}
$$

If $5 \leq p_{j} \leq 19$, we have $\frac{a}{p_{j}-1-a}=\frac{2}{p_{j}-3} \leq 1$. If $p_{j}>19$, we have $\frac{a}{p_{j}-1-a}=\frac{3}{p_{j}-4}<1$. Therefore we can conclude that if $r k\left(\mathbb{Z}^{n}\right)^{P_{j}} \leq n-2$, then $d(\Gamma) \leq n$. By Cauchy's Theorem 
[9, Page 93], $P_{j}$ has an element $x \in P_{j}$ with order $p_{j}$. Let $C_{p_{j}}$ be a cyclic subgroup of $P_{j}$ generated by $x$. Consider $\left(\mathbb{Z}^{n}\right)^{C_{P_{j}}}$, where $C_{P_{j}}$ acts faithfully on $\mathbb{Z}^{n}$ via $\left.\rho\right|_{C_{P_{j}}}: C_{P_{j}} \rightarrow$ $G L_{n}(\mathbb{Z})$. By [7, Section 73], the degree of a faithful indecomposable $C_{p_{j}}$-representation is either $p_{j}-1$ or $p_{j}$. If the degree is $p_{j}-1$, then it has trivial fix point set. If the degree is $p_{j}$, then the fix point set is 1-dimensional. Observe that $r k\left(\mathbb{Z}^{n}\right)^{C_{p_{j}}}$ has maximum value when $\left.\rho\right|_{C_{P_{j}}}$ is a direct sum of one faithful indecomposable sub-representation and all others are trivial sub-representations. Therefore $r k\left(\mathbb{Z}^{n}\right)^{C_{p_{j}}} \leq n-p_{j}+1 \leq n-4$. Hence we have $r k\left(\mathbb{Z}^{n}\right)^{P_{j}} \leq n-4$. Therefore we can conclude $d(\Gamma) \leq n$.

(ii): By [1, Theorem A], we can assume $G$ is not a $p$-group. By [11, Theorem A], we have

$$
d(G) \leq \max _{1 \leq i \leq k} d\left(P_{i}\right)+1
$$

where $P_{i}$ is the Sylow $p_{i}$-subgroup of $G$ for $i=1, \ldots, k$. If $\max _{1 \leq i \leq k} d\left(P_{i}\right) \neq d\left(P_{1}\right)$, then by part (i), we have $d(\Gamma) \leq n$. Therefore we assume $\max _{1 \leq i \leq k} d\left(P_{i}\right)=d\left(P_{1}\right)$. We can consider the lattice part as a $\mathbb{Z} P_{1}$-module. Since $P_{1}$ is a Sylow 3 -subgroup, by [1, Theorem A], we have $d\left(P_{1}\right)+r k_{P_{3}}\left(\mathbb{Z}^{n}\right) \leq n$. Hence we can conclude that $d(\Gamma) \leq d\left(P_{3}\right)+r k_{P_{3}}\left(\mathbb{Z}^{n}\right)+1=$ $n+1$.

Theorem C Let $\Gamma$ be an n-dimensional Bieberbach group with 2-generated holonomy group. Then $d(\Gamma) \leq n$.

Proof Let $G$ be the holonomy group of $\Gamma$. Let $x$ and $y$ be the generators of $G$. They have order $a$ and $b$ respectively. If either $a=1$ or $b=1$, then $G$ is a cyclic group. By [8, Theorem 5.7] and Theorem A, $d(\Gamma) \leq n$. Next, consider cases where $a \geq 3$ or $b \geq 3$. It is sufficient to consider only the case where $a \geq 3$. By Remark 2.1, let $\Gamma=\left\langle\iota\left(e_{1}\right), \ldots, \iota\left(e_{n}\right), \alpha, \beta\right\rangle$, where $e_{1}, \ldots, e_{n}$ are the standard basis for $\mathbb{Z}^{n}, p(\alpha)=x$ and $p(\beta)=y$. Define $\Gamma^{\prime}=\left\langle\iota\left(e_{1}\right), \ldots, \iota\left(e_{n}\right), \alpha\right\rangle$. Notice that $\Gamma^{\prime}$ is an $n$-dimensional Bieberbach subgroup of $\Gamma$ with holonomy group $C_{a}$. Since $a \geq 3$, by Theorem A, $d\left(\Gamma^{\prime}\right) \leq n-1$. Hence we have $d(\Gamma) \leq n$. Finally, we assume $a=b=2$. Consider element $x y \in G$. Since $G$ is finite, $x y$ has finite order. If $x y$ is of order 1 (i.e. $x y=1$ ), then $x=y$. So $G \cong C_{2}$. By [8, Theorem 5.7], $d(\Gamma) \leq n$. If $x y$ is of order 2 (i.e. $x y x y=1$ ), then $x y=y x$. Hence $G \cong C_{2} \times C_{2}$. By [8, Theorem 5.7], we have $d(\Gamma) \leq n$. Lastly, we assume $x y$ is of order $k$, where $k \geq 3$. We can rewrite the generating set of $\Gamma$ to be $\left\{\iota\left(e_{1}\right), \ldots, \iota\left(e_{n}\right), \alpha \beta, \beta\right\}$. Define $\Gamma^{\prime \prime}=\left\langle\iota\left(e_{1}\right), \ldots, \iota\left(e_{n}\right), \alpha \beta\right\rangle$, which is an $n$-dimensional Bieberbach subgroup of $\Gamma$ with holonomy group isomorphic to $C_{k}$. By Theorem A, $d\left(\Gamma^{\prime \prime}\right) \leq n-1$. Therefore $d(\Gamma) \leq n$.

By $[1,8]$ and the three main theorems in this paper, the Conjecture 1.1 is still open for certain cases of holonomy group where the minimal number of generators has at least three elements. For example, the case where the holonomy group is a 2-group or the order of holonomy group is even. By Corollary 4.1 , the corresponding $n$-dimensional Bieberbach group can be generated by $2 n$ elements. Another case is when the order of holonomy group is odd and divisible by 3 . In this case, by Theorem $\mathrm{B}$, the corresponding $n$-dimensional Bieberbach group can be generated by $n+1$ elements. In order to prove this conjecture fully, we believe further study is needed in the key case where the holonomy group is a 2-group.

Acknowledgements I would like to thank my supervisor Dr. Nansen Petrosyan for his help and guidance.

Open Access This article is distributed under the terms of the Creative Commons Attribution 4.0 International License (http://creativecommons.org/licenses/by/4.0/), which permits unrestricted use, distribution, and reproduction in any medium, provided you give appropriate credit to the original author(s) and the source, provide a link to the Creative Commons license, and indicate if changes were made. 


\section{References}

1. Adem, A., Dekimpe, K., Petrosyan, N., Putrycz, B.: On generators of crystallographic groups and actions on flat orbifolds. J. Group Theory 15(4), 553-561 (2012)

2. Adem, A., Ge, J., Pan, J., Petrosyan, N.: Compatible actions and cohomology of crystallographic groups. J. Algebra 320(1), 341353 (2008)

3. Aschbacher, M., Guralnick, R.: Some applications of the first cohomology group. J. Algebra 90, 446-460 (1984)

4. Brown, K.S.: Cohomology of Groups. Springer, New York (1982)

5. Crystallographic AlgoRithms And Tables (CARAT) Lehrstuhl B fur Mathematik, Aachen (2006). http:// wwwb.math.rwth-aachen.de/carat/

6. Charlap, L.S.: Bieberbach Groups and Flat Manifolds. Universitext. Springer, Berlin (1986)

7. Curtis, W.C., Reiner, I.: Representation Theory of Finite Groups and Associative Algebras. Wiley, New York (1962)

8. Dekimpe, K., Penninckx, P.: On the number of generators of a Bieberbach group. Commun. Algebra 37(12), 4332-4345 (2009)

9. Dummit, D.S., Foote, R.M.: Abstract Algebra, Third edn. Wiley, Hoboken (2003)

10. Goncalbes, D.L., Vieira, J.P.: Infra-abelian groups and free actions of finite groups on the $n$-torus. Commun. Algebra 30(6), 2791-2803 (2002)

11. Guralnick, R.M.: On the number of generators of a finite group. Arch. Math. 53, 521-523 (1989)

12. Hiller, H., Sah, C.H.: Holonomy of flat manifolds with $b_{1}=0$. Q. J. Math. 37, 177-187 (1986)

13. Ribenboim, P.: Classical Theory of Algebraic Numbers. Springer, Heidelberg (2001)

14. Reiner, I.: Integral representations of cyclic groups of prime order. Proc. Am. Math. Soc. 8, 142-146 (1957)

15. Szczepanski, A.: Geometry of Crystallographic Groups. World Scientific, Singapore (2012)

16. Tahara, K.I.: On the finite subgroups of GL(3, Z). Nagoya Math. J. 41, 169-209 (1971) 